\title{
La presencia de células en anillo de sello en la biopsia endoscópica no es un buen predictor para el diagnóstico de carcinoma gástrico de células en anillo de sello
}

${ }^{1}$ Hospital Sótero del Río. Pontificia Universidad Católica de Chile. Santiago, Chile.

Recibido el 23 de septiembre de 2017, aceptado para publicación el 25 de noviembre de 2017

Correspondencia a: Dr. Marco Ceroni V. marco.ceroni@gmail.com

\author{
Marco Ceroni V. ${ }^{1}$, Enrique Norero M. ${ }^{1}$, Cristián Martínez B. ${ }^{1}$, Ricardo Mejia M. ${ }^{1}$, \\ Rodrigo Muñoz C. ${ }^{1}$, Gloria Aguayo B. ${ }^{1}$, Paulina González C. ${ }^{1}$ y Alfonso Díaz F. ${ }^{1}$
}

The presence of signet-ring cell in endoscopic biopsy is not a good predictor for the diagnosis of signet-ring cell carcinoma of the stomach

Introduction: Signet-ring cell carcinoma (SRCC) of the stomach is a histopathological type that has less response to chemotherapy and worse prognosis in patients with advanced gastric cancer, than other types of gastric carcinomas. Diagnostic value of the presence of signet-ring cells (SRC) in the endoscopic biopsy for the diagnosis of SRCC of the stomach, are unknown. Objectives: To calculate the diagnostic values of the presence of SRC in endoscopic biopsy for the diagnosis of SRCC of the stomach in a definitive surgical specimen biopsy. Materials and Methods: Retrospective diagnostic test study to determine the value of the presence of SRC in the endoscopic biopsy for the diagnosis of SRCC of the stomach in the surgical specimen biopsy. Inclusion criteria: Patients who underwent gastric surgery between 1996-2016. We calculated positive and negative predictive values (PPV and NPV), sensitivity, specificity, and positive and negative likelihood ratio (LR+ and LR-) of the presence of SRC in the endoscopic biopsy that predicts the diagnosis of SRCC of the stomach in the definitive biopsy. Confidence intervals (CI) of 95\% were defined. Results: The diagnostic values of the presence of SRC in endoscopic biopsy to diagnose SRCC of the stomach in the surgical specimen biopsy were: PPV of 56.1\% (95\% CI, 47.8-64.1\%), NPV of 91.3\% (95\% CI, 89-93.1\%), sensitivity of 55.7\% (95\% CI, 47.4-63.7\%), specificity of 91.4\% (95\% CI, 89.1-93.3\%), LR+ of 6.5 (95\% CI, 4.9-8.6) and LR- of 0.48 (95\% CI, 0.4-0.6), a positive post-test probability of $56.1 \%$ (95\% CI, 47.8-64.1\%), and a negative post-test probability of $8.7 \%$ (95\% CI, $6.9-11 \%)$. Conclusions: The presence of SRC in the endoscopic biopsy is not sufficient to diagnose SRCC of the stomach. The absence of SRC in the endoscopic biopsy has a high negative predictive value.

Key words: gastric cancer; signet-ring cell carcinoma of the stomach; signet-ring cell.

\section{Resumen}

Introducción: El carcinoma gástrico de células en anillo de sello (CGCAS) es un tipo histopatológico, que tiene menor respuesta a la quimioterapia $(\mathrm{QT})$ y un peor pronóstico en los pacientes con cáncer gástrico (CG) avanzado. Se desconoce los valores diagnósticos de la presencia de células en anillo de sello (CAS) en la biopsia endoscópica, para el diagnóstico de CGCAS. Objetivo: Determinar los valores diagnósticos de la presencia de CAS en la biopsia endoscópica para el diagnóstico de CGCAS en la biopsia de la pieza operatoria. Material y Método: Estudio retrospectivo de pruebas diagnósticas. Se incluyeron los pacientes con CG operados en forma consecutiva entre 1996-2016. Se calculó los valores diagnósticos de la presencia de CAS en la biopsia endoscópica para el diagnóstico de CGCAS en la biopsia definitiva. Se utilizaron intervalos de confianza (IC) del 95\%. Resultados: Se incluyeron 851 pacientes. Un 16,3\% tuvieron CAS en la biopsia endoscópica y la prevalencia de CGCAS fue de 16,4\%. Los valores diagnósticos de la presencia de CAS de la biopsia endoscópica para el diagnóstico de CGCAS fueron: Valor predictivo positivo (VPP) de 56,1\% (IC 95\%, 47,8-64,1\%); Valor predictivo negativo (VPN) de 91,3\% (IC 95\%, 89-93,1\%); sensibilidad de 55,7\% (IC 95\%, 47,4-63,7\%); especificidad de 91,4\% (IC 95\%, 89,1\%-93,3\%); Likelihood 
ratio (LR) positivo de 6,5 (IC 95\%, 4,9-8,6); LR negativo de 0,48 (IC 95\%, 0,4-0,6); probabilidad post-test positivo fue de $56,1 \%$ (IC 95\%, 47,8-64,1\%) y probabilidad post-test negativo fue de 8,7\% (IC 95\%, 6,9$11 \%$ ). Conclusiones: La presencia de CAS en la biopsia endoscópica es insuficiente para el diagnóstico de un CGCAS. La ausencia de CAS en la biopsia endoscópica tiene un alto valor predictivo negativo. Palabras clave: cáncer gástrico; carcinoma gástrico de células en anillo de sello; células en anillo de sello.

\section{Introducción}

El carcinoma gástrico de células en anillo de sello (CGCAS) es un tipo histopatológico de cáncer gástrico ( $\mathrm{CG}$ ), definido por la organización mundial de la salud (OMS) como la presencia de más de $50 \%$ de células aisladas o en grupo con mucina intracitoplasmática ${ }^{1}$. Tiene una incidencia estimada entre $3-39 \%$, la que ha ido aumentado con el tiempo en los países occidentales y en Chile, en comparación a los otros tipos histopatológicos de $\mathrm{CG}^{2-12}$. El pronóstico oncológico es menor en pacientes que tienen un CGCAS avanzado, con mayor capacidad de infiltración de la pared gástrica, siendo sus principales vías de diseminación la linfática y a través del peritoneo ${ }^{11}$. Debido a que es un cáncer con menor respuesta a la quimioterapia, en comparación con otros tipos histopatológicos, la cirugía es la indicación primaria ${ }^{12}$.

En nuestro centro, hemos observado que una cantidad importante de los pacientes con CG con presencia de CAS en la biopsia endoscópica preoperatoria, no tienen CGCAS en la biopsia definitiva de la pieza operatoria. Debido a que ésta información tiene implicancias en las decisiones terapéuticas, realizamos un estudio de pruebas diagnósticas para determinar el valor de la presencia de CAS en la biopsia endoscópica, para el diagnóstico de CGCAS de la pieza operatoria.

\section{Objetivos}

Calcular los valores diagnósticos de la presencia de CAS en la biopsia endoscópica para el diagnóstico de CGCAS en la biopsia de la pieza operatoria.

Calcular la concordancia entre la biopsia endoscópica y la biopsia definitiva de la pieza operatoria para la presencia de CAS.

\section{Material y Método}

Estudio retrospectivo de pruebas diagnósticas para calcular el valor de la presencia de CAS en la biopsia endoscópica en pacientes con $\mathrm{CG}$, para el diagnóstico de CGCAS de la biopsia de la pieza operatoria. Se incluyeron todos los pacientes con CG tratados con gastrectomía o con cirugía endoscópica, en forma consecutiva entre 1996-2016 del Complejo Asistencial Hospital Sótero del Rio. Se consideró como gold standard el informe definitivo de la pieza operatoria. El informe de anatomía patológica de la biopsia endoscópica fue realizado en forma independiente y no influyó en el informe de la biopsia de la pieza operatoria. En todos los pacientes se utilizó la clasificación histopatológica de la $\mathrm{OMS}^{1}$. En este estudio no se realizó una nueva revisión de las biopsias, para evaluar los resultados de la práctica habitual de nuestro centro, en el período incluido. Las biopsias endoscópicas fueron clasificadas según la presencia o ausencia de CAS, a su vez, las biopsias de las piezas operatorias fueron clasificadas si tuvieron el diagnóstico o no de CGCAS. Se excluyeron los pacientes con cirugía de urgencia que no tenían biopsia endoscópica previo a la cirugía, los pacientes que no tenían disponible el informe de la biopsia endoscópica y otros tipos de tumores gástricos (linfomas, displasias, tumores estromales gastrointestinales y linfomas). Se calculó el valor predictivo positivo (VPP), el valor predictivo negativo (VPN), sensibilidad, especificidad, likelihood ratio (LR) positivo y LR negativo de la presencia de CAS en la biopsia endoscópica para el diagnóstico de CGCAS en la biopsia definitiva. Se calculó los intervalos de confianza (IC) de 95\%. Se utilizó el índice de Kappa y la tabla de Landis-Koch ${ }^{13}$ para determinar la concordancia de la presencia de CAS, entre la biopsia endoscópica y la biopsia definitiva de la pieza operatoria. Para la etapificación patológica, se utilizó el TNM $7^{\text {a }}$ edición $^{14}$. Se realizó el análisis estadístico con el programa GraphPad Prism versión 5.0. El estudio fue aprobado por el comité de ética de nuestra institución.

\section{Resultados}

Constituyen el estudio 851 pacientes con diagnóstico de $\mathrm{CG}$, los cuales, el 100\% tienen informe de la biopsia endoscópica e informe definitivo de 


\section{ARTÍCULO ORIGINAL}

la pieza operatoria. Fueron de sexo masculino 567 $(66,6 \%)$ pacientes, con una edad promedio de 64,4 años (DE 12,5), a $551(63,4 \%)$ pacientes se les realizó una gastrectomía total, a 689 (79,2\%) la cirugía fue con intención curativa, $153(17,9 \%)$ pacientes tuvieron el diagnóstico de un $\mathrm{CG}$ incipiente. En $140(16,4 \%)$ pacientes se realizó el diagnóstico definitivo de CGCAS en la pieza operatoria, de los cuales, 56\% tenían CAS en el informe de la biopsia endoscópica (Tabla 1).

Los valores diagnósticos de la biopsia endoscópica para el diagnóstico de CGCAS en la biopsia de la pieza operatoria fueron: VPP de 56,1\% (IC 95\%, 47,8-64,1\%); VPN de 91,3\% (IC 95\%, 89-93,1\%); sensibilidad de 55,7\% (IC 95\%, 47,4-63,7\%); especificidad de 91,4\% (IC 95\%, 89,1\%-93,3\%); LR positivo de 6,5 (IC 95\%, 4,9-8,6); LR negativo de 0,48 (IC 95\%, 0,4-0,6); probabilidad post-test positivo de $56,1 \%$ (IC $95 \%, 47,8-64,1 \%$ ), en otras palabras, la presencia de CAS en el informe de la biopsia endoscópica aumenta la probabilidad de tener un CGCAS de $16,4 \%$ (prevalencia) a 56,1\% y una probabilidad post-test negativa de $8,7 \%$ (IC $95 \%, 6,9-11 \%$ ) o la ausencia de CAS en el informe de la biopsia endoscópica disminuye la probabilidad de tener un CGCAS de $16,4 \%$ a $8,7 \%$. Se realizaron los cálculos de pruebas diagnósticas separando los pacientes con CG incipiente y avanzado (Tablas 2 y 3). No se encontró diferencias en los valores de las pruebas diagnósticas comparando los pacientes con CG avanzado e incipiente (Tabla 4). El índice de Kappa fue de 0,46 (IC 95\%, 0,36-0,52), lo que equivale a una concordancia baja a moderada entre las 2 biopsias.

Tabla 1. Tabla de contingencia con la distribución de los resultados de las biopsias endoscópicas y definitivas de las piezas operatorias, para la presencia/ausencia de CAS y de la presencia/ausencia de CGCAS

\begin{tabular}{|lccc|}
\hline & $\begin{array}{c}\text { Biopsia de la pieza operatoria } \\
\text { positiva para CGCAS }\end{array}$ & $\begin{array}{c}\text { Biopsia de la pieza operatoria } \\
\text { negativa para CGCAS }\end{array}$ & $\begin{array}{c}\text { Total } \\
\text { Biopsia endoscópica positiva para CAS }\end{array}$ \\
Biopsia endoscópica negativa para CAS & 78 & 61 & 139 \\
Total & 62 & 650 & 712 \\
\hline
\end{tabular}

Tabla 2. Tabla de contingencia con la distribución de los resultados de las biopsias endoscópicas y definitivas de las piezas operatorias para pacientes con CG incipiente, para la presencia/ausencia de CAS y de la presencia/ausencia de CGCAS

\begin{tabular}{|lccc|}
\hline & $\begin{array}{c}\text { Biopsia de la pieza operatoria } \\
\text { positiva para CGCAS }\end{array}$ & $\begin{array}{c}\text { Biopsia de la pieza operatoria } \\
\text { negativa para CGCAS }\end{array}$ & $\begin{array}{c}\text { Total } \\
\text { Biopsia endoscópica positiva para CAS }\end{array}$ \\
Biopsia endoscópica negativa para CAS & 13 & 124 & 21 \\
Total & 8 & 132 & 132 \\
\hline
\end{tabular}

Tabla 3. Tabla de contingencia con distribución de los resultados de las biopsias endoscópicas y definitivas de las piezas operatorias para pacientes con CG avanzado, para la presencia/ausencia de CAS y presencia/ausencia de CGCAS

\begin{tabular}{|lccc|}
\hline & $\begin{array}{c}\text { Biopsia de la pieza operatoria } \\
\text { positiva para CGCAS }\end{array}$ & $\begin{array}{c}\text { Biopsia de la pieza operatoria } \\
\text { negativa para CGCAS }\end{array}$ & $\begin{array}{c}\text { Total } \\
\text { Biopsia endoscópica positiva para CAS }\end{array}$ \\
Biopsia endoscópica negativa para CAS & 70 & 53 & 123 \\
Total & 54 & 526 & 580 \\
\hline
\end{tabular}


Tabla 4. Valores diagnósticos para todos los pacientes del estudio y separado por CG avanzado e incipiente

\begin{tabular}{|lccc|}
\hline & CG avanzado + incipiente & CG Avanzado & CG Incipiente \\
Sensibilidad & $55,7 \%$ & $56,5 \%$ & $61,9 \%$ \\
Especificidad & $91,4 \%$ & $90,8 \%$ & $93,9 \%$ \\
VPP & $56,1 \%$ & $56,9 \%$ & $61,9 \%$ \\
VPN & $91,3 \%$ & $90,7 \%$ & $93,9 \%$ \\
LR+ & 6,49 & 6,17 & 10,21 \\
LR- & 0,48 & 0,48 & 0,41 \\
Probabilidad post-test + & $56 \%$ & $54,7 \%$ & $66,7 \%$ \\
Probabilidad post-test - & $8,7 \%$ & $8,6 \%$ & $7,4 \%$ \\
\hline
\end{tabular}

\section{Discusión}

El CG es una enfermedad heterogénea con variación epidemiológica, histopatológica y del pronóstico ${ }^{2-6,15}$. En la última década, el tratamiento del CG avanzado ha tenido un gran desarrollo, se han individualizado las terapias, al incorporar la QT, la radioterapia y la terapia inmunológica. La cirugía sigue siendo la parte fundamental del tratamiento con intención curativa y también, en algunos pacientes que requieren paliación o cirugía de conversión ${ }^{16}$. Por esta razón los algoritmos de tratamiento y los paradigmas de las décadas pasadas han ido cambiado y para la mayoría de los pacientes, sin metástasis a distancia, la QT es parte integral del tratamiento sistémico ${ }^{17,18}$. Sin embargo, el CGCAS avanzado es un subtipo histopatológico, que determina un cambio de conducta debido a su mal pronóstico ${ }^{11,19}$ y su baja respuesta a la $\mathrm{QT}^{12,20}$. En estos casos la cirugía es la indicación primaria.

En pacientes con CG, la presencia de CAS en la biopsia endoscópica preoperatoria, se ha considerado por mucho tiempo como el elemento principal para el diagnóstico de CGCAS y tomar decisiones en el tratamiento, sin embargo, en una cantidad importante $(43,8 \%)$ de nuestros pacientes con CAS en la biopsia endoscópica preoperatoria, no se confirmó el diagnóstico de CGCAS. Esto pone en duda el valor diagnóstico de la presencia de CAS de la biopsia endoscópica para el diagnóstico de CGCAS, como también, debilita la conducta terapéutica de indicar la cirugía sin una QT neoadyuvante, ya que pueden corresponder a otros subtipos histológicos que se benefician de un tratamiento sistémico perioperatorio.

Debido a que la presencia de CAS en la biopsia endoscópica tiene bajo VPP $(56,1 \%)$ para el diag- nóstico definitivo de CGCAS, no se pueden tomar decisiones terapéuticas con esta información. Esto concuerda con otros estudios reportados, en que la biopsia endoscópica difiere de la biopsia definitiva de la pieza operatoria, con rangos que varían entre el 13 y el $30 \%{ }^{21-25}$. Sólo en un estudio, se demostró que la presencia de CAS biopsia endoscópica es un buen predictor de CGCAS, este resultado se debe a que hay una revisión y reevaluación de las biopsias endoscópicas de una cantidad importante de pacientes ${ }^{26}$. En el presente estudio no realizamos revisión de las biopsias, para tener la información de la conducta habitual de nuestro centro.

Esta diferencia en los informes de las biopsias se puede deber a varios factores, como son el sesgo en la toma de las muestras endoscópicas, tomar una cantidad insuficiente de muestras del cáncer, obtener un escaso volumen de las muestras de la biopsia y a la existencia de errores en la preparación del tejido en el laboratorio de anatomía patológica ${ }^{22}$. Las dos opciones para mejorar la precisión del diagnóstico endoscópico y poder tomar decisiones terapéuticas correctas son: 1) Revisar o repetir las biopsias endoscópicas en los pacientes con CG avanzado en que se observen $\mathrm{CAS}^{26}$. Se ha reportado que a medida que se aumenta la cantidad de endoscopias diagnósticas, la concordancia con la biopsia definitiva aumenta en forma significativa, con una endoscopia se obtiene una concordancia entre las biopsias de $70 \%$, con 2 endoscopias de $95 \%$ y con 3 , de $98 \%{ }^{22}$. Sin embargo, esta conducta aumenta los costos y los riesgos del procedimiento para los pacientes; 2) Tomar durante la endoscopia, al menos 7 muestras del cáncer. Esta conducta obtiene el diagnóstico histológico correcto en todos los pacientes estudiados, sin embargo, esto no siempre es posible, por ejemplo, como son los pacientes con CG incipiente, 
debido a que presentan lesiones pequeñas y de bajo volumen $^{27}$.

Las alternativas en los pacientes con diagnóstico de CG con presencia de CAS, en que se decide no repetir la endoscopia y tienen menos de 7 biopsias son dos: 1) Indicar la QT neoadyuvante en todos los pacientes con CG avanzado, sabiendo que un 16,4\% de los pacientes tendrán un CGCAS en la biopsia definitiva y luego indicar la cirugía; 2) Indicar la secuencia terapéutica según el riesgo del paciente de tener complicaciones quirúrgicas y de la posibilidad de llegar a la adyuvancia. Para los pacientes con alto riesgo de presentar complicaciones postoperatorias (presencia de cáncer de la unión gastroesofágica, cáncer de píloro con infiltración duodenal o invasión de varios órganos), es preferible indicar primero QT y posteriormente cirugía. Y al resto de los pacientes, realizar la cirugía y luego, algún tratamiento adyuvante. La justificación de esta conducta es no perder el beneficio de la QT debido a posibles complicaciones postoperatorias y que los pacientes la reciban en un estado nutricional óptimo y sin retrasos.

Con respecto a los pacientes con CGCAS incipientes, la literatura en forma consistente ha demostrado que son un grupo de buen pronóstico ${ }^{15,28,29}$ y tienen un comportamiento similar al grupo con histología bien diferenciada, por lo que pueden ser tratados igual que un paciente con CG incipiente bien diferenciado. Por lo que la presencia de CAS no presenta un problema para la toma de decisiones. Al igual que el resto de los $\mathrm{CG}$ incipientes los factores determinantes de riesgo de compromiso ganglionar son la invasión linfovascular, la invasión de la submucosa y el tamaño mayor a $2 \mathrm{~cm}^{30-32}$.

Un método novedoso, que aún se encuentra en etapa de investigación, es la microespectrometría confocal Raman, el cual se utiliza para diferenciar los subtipos histológicos de CG. Es una técnica que utiliza luz láser sobre un objeto y según los componentes moleculares de la materia de estudio, produce modificaciones espectrales altamente específicas. Esta técnica tiene la capacidad de diferenciar tipos histológicos con excelente especificidad (entre adenocarcinoma tubular y papilar del CGCAS) ${ }^{33}$.

En resumen, la presencia de CAS en la biopsia endoscópica no es un buen predictor para el diagnóstico definitivo de CGCAS, por lo que las decisiones de tratamiento de los pacientes con $\mathrm{CG}$ avanzado con CAS en la biopsia endoscópica deben ser realizadas según la posibilidad de llegar a la adyuvancia, en base a su riesgo de presentar complicaciones postoperatorias. Se pueden mejorar los resultados de la biopsia endoscópica, repitiendo las endoscopias con biopsias con presencia de CAS y aumentando a 7 la cantidad de muestras endoscópicas del cáncer.

\section{Responsabilidades éticas}

Protección de personas y animales. Los autores declaran que para esta investigación no se han realizado experimentos en seres humanos ni en animales.

Confidencialidad de los datos. Los autores declaran que han seguido los protocolos de su centro de trabajo sobre la publicación de datos de pacientes.

Derecho a la privacidad y consentimiento informado. Los autores han obtenido el consentimiento informado de los pacientes y/o sujetos referidos en el artículo. Este documento obra en poder del autor de correspondencia.

\section{Conflicto de intereses}

Los autores declaran no tener ningún conflicto de intereses.

\section{Bibliografía}

1. Watanabe H, Jass JR, Sobin LH. Histological typing of oesophageal and gastric tumours. In: WHO International Histological Classification of Tumors. 1990 2nd ed. Springer-Verlag, Berlín.

2. Calderón M, Csendes A, Ospina C, Lara A, Hodgson F. Evolución del cáncer gástrico en 30 años 1975-2005. Rev Chil Cir. 2007;59:366-9.

3. Covacevich S, Jarufe N, Barrera A. Análisis de la efectividad diagnóstica de la endoscopia, radiología y cirugía en cáncer gástrico. Rev Chil Cir. 1997;49: 650-4.

4. Venturelli F, Cárcamo C, Venturelli A, Cárcamo M, Born M, Jara C, et al. Análisis de sobrevida del cáncer gástrico en anillo de sello según profundidad y compromiso linfonodal. Rev Chil Cir. 2008;60:398-402.

5. Csendes A, Smok G, Medina E, Salgado I, Rivera R, Quintral M. Características evolutivas del cáncer gástrico 1958-1990. Rev Med Chile 1992;120:36-42.

6. Tapia O, Gutiérrez V, Roa J, Manterola
C, Villaseca M, Araya J. Carcinoma de células en anillo de sello gástrico: descripción clínico-morfológica y valor pronóstico. Rev Chil Cir. 2010;5:458-64.

7. Clark CJ, Thirlby RC, Picozzi V Jr, Schembre DB, Cummings FP, Lin E. Current problems in surgery: gastric cancer. Curr Probl Surg. 2006;43: 566-670.

8. Henson DE, Dittus C, Younes M, Nguyen H, Albores-Saavedra J. Differential trends in the intestinal and diffuse types 
of gastric carcinoma in the United States 1973-2000: increase in the signet ring cell type. Arch Pathol Lab Med. 2006;128:765-70.

9. Wu H, Rusiecki JA, Zhu K, Potter J, Devesa SS. Stomach carcinoma incidence patterns in the United States by histologic type and anatomic site. Cancer Epidemiol Biomarkers Prev. 2009;18:1945-52.

10. Cunningham SC, Kamangar F, Kim MP, Hammoud S, Haque R, Maitra A, et al. Survival after gastric adenocarcinoma resection: eighteen-year experience at a single institution. J Gastrointest Surg. 2005;9:718-25.

11. Piessen G, Messager M, Leteurtre E, Jean-Pierre T, Mariette C. Signet ring cell histology is an independent predictor of poor prognosis in gastric adenocarcinoma regardless of tumoral clinical presentation. Ann Surg. 2009;250:878-87.

12. Messager M, Lefevre JH, Pichot-Delahaye V, Souadka A, Piessen G, Mariette C. FREGAT working group-FRENCH. Impact of perioperative chemotherapy on survival in patients with gastric signet ring cell adenocarcinoma: a multicentric comparative study. Ann Surg. 2011;254:684-93.

13. Landis J, Koch G. The measurement of observer agreement for categorical data. Biometrics 1977;33:159-74.

14. Edge SB, Byrd Dr, Compton CC. editors. AJCC Cancer Staging Manual. 7th ed New York, NY: Springer; 2010.

15. Pernot S, Voron T, Perkins G, LagorcePages C, Berger A, Taieb J. Signet-ring cell carcinoma of the stomach: Impact on prognosis and specific therapeutic challenge. World J Gastroenterol. 2015;21:11428-38.

16. Yoshida K, Yamaguchi K, Okumura N, Tanahashi T, Kodera Y. Is conversion therapy posible in stage IV gastric cancer: the proposal of new biological categories of classification. Gastric Cancer 2016;19:329-38.

17. Cunningham D, Allum WH, Stenning SP, Thompson JN, Van de Velde CJ,
Nicholson M, et al. MAGIC Trial Participants. Perioperative chemotherapy versus surgery alone for resectabe gastroesophageal cancer. NEJM 2006;355:11-20.

18. Ychou M, Boige V, Pignon JP, Conroy T, Bouché $\mathrm{O}$, Lebreton $\mathrm{G}$, et al. Perioperative chemotherapy compared with surgery alone for resectable gastroesophageal adenocarcinoma: an FNCLCC and FFCD multicenter phase III trial. J Clin Oncol. 2011;29:1715-21.

19. Lu M, Yang Z, Feng Q, Yu M, Zhang $\mathrm{Y}, \mathrm{Mao} \mathrm{C}$, et al. The characteristics and prognostic value of signet ring cell histology in gastric cancer: A retrospective cohort study of 2199 consecutive patients. Medicine (Baltimore). 2016;95:e4052.

20. Li C, Kim S, Lai JF, Hyung WJ, Choi $\mathrm{WH}$, Choi SH, et al. Advanced gastric carcinoma with signet ring cell histology. Oncology 2007;72:64-8.

21. Jónasson L, Hallgrímsson J, Olafsdóttir G. Gastric carcinoma: correlation of diagnosis based on biopsies and resection specimens with reference to the Laurén classification. APMIS. 1994;102:711-5.

22. Golembeski CP, Genta RM. Signetring cell carcinoma in gastric biopsies: expecting the unexpected. J Clin Pathol. 2013;66:136-9.

23. Flucke U, Mönig SP, Baldus SE, Zirbes TK, Bollschweiler E, Thiele J, et al. Differences between biopsy- or specimenrelated Laurén and World Health Organization classification in gastric cancer. World J Surg. 2002;26:137-40.

24. Hansson LE, Lindgren A, Nyrén O. Can endoscopic biopsy specimens be used for reliable Laurén classification of gastric cancer? Scand J Gastroenterol. 1996;31:711-5.

25. Matsubara $Y$, Yanai $H$, Ishiguro $K$, Ryozawa S, Okazaki Y, Matsui N, et al. Clinical interpretation of the histological typing of gastric cancer using endoscopic forceps biopsy. Hepatogastroenterology 2004;51:285-8.

26. Piessen G, Amielh D, Messager M,
Vinatier E, Leteurtre E, Triboulet JP, et al. Is pretreatment endoscopic biopsy a good predictor of signet ring cell histology in gastric carcinoma? World J Surg. 2012;36:346-54

27. Graham DY, Schwartz JT, Cain GD, Gyorkey F. Prospective evaluation of biopsy number in the diagnosis of esophageal and gastric carcinoma. Gastroenterology 1982;82:228-31.

28. Gronnier C, Messager M, Robb WB, Thiebot T, Louis D, Luc G, Piessen G, et al. FREGAT working group-FRENCH. Is the negative prognostic impact of signet ring cell histology maintained in early gastric adenocarcinoma? Surgery 2013;154:1093-9.

29. Kunisaki C, Shimada H, Nomura M, Matsuda G, Otsuka Y, Akiyama H. Therapeutic strategy for signet ring cell carcinoma of the stomach. Br J Surg. 2004;91:1319-24.

30. Guo CG, Zhao DB, Liu Q, Zhou ZX, Zhao P, Wang GQ, et al. Risk Factors for Lymph Node Metastasis in Early Gastric Cancer with Signet Ring Cell Carcinoma. J Gastrointest Surg. 2015;19:1958-65.

31. Park JM, Kim SW, Nam KW, Cho YK, Lee IS, Choi MG, et al. Is it reasonable to treat early gastric cancer with signet ring cell histology by endoscopic resection? Analysis of factors related to lymph-node metastasis. Eur J Gastroenterol Hepatol. 2009;21:1132-5.

32. Kim HM, Pak KH, Chung MJ, Cho JH, Hyung WJ, Noh SH, et al. Early gastric cancer of signet ring cell carcinoma is more amenable to endoscopic treatment than is early gastric cancer of poorly differentiated tubular adenocarcinoma in select tumor conditions. Surg Endosc. 2011;25:3087-93.

33. Chih-Wei Hsu, Chia-Chi Huang, JengHorng Sheu, Chia-Wen Lin, Lien-Fu Lin, Jong- Shiaw Jin, et al. Novel Method for Differentiating Histological Types of Gastric Adenocarcinoma by Using Confocal Raman Microspectroscopy. PLoS One. 2016;11. 